\title{
Applying He's Variational Iteration Method for Solving Differential-Difference Equation
}

\author{
Ahmet Yildirim \\ Department of Mathematics, Faculty of Science, Ege University, 35100 Bornova-İzmir, Turkey
}

Correspondence should be addressed to Ahmet Yıldırım, ahmety@mail.ege.edu.tr

Received 31 January 2008; Revised 1 April 2008; Accepted 14 May 2008

Recommended by Oleg Gendelman

We extend He's variational iteration method (VIM) to find the approximate solutions for nonlinear differential-difference equation. Simple but typical examples are applied to illustrate the validity and great potential of the generalized variational iteration method in solving nonlinear differentialdifference equation. The results reveal that the method is very effective and simple. We find the extended method for nonlinear differential-difference equation is of good accuracy.

Copyright ( 92008 Ahmet Yıldırım. This is an open access article distributed under the Creative Commons Attribution License, which permits unrestricted use, distribution, and reproduction in any medium, provided the original work is properly cited.

\section{Introduction}

In recent years, some promising approximate analytical solutions are proposed, such as expfunction method [1], homotopy perturbation method [2-11], and variational iteration method (VIM) [12-25]. The variational iteration method is the most effective and convenient one for both weakly and strongly nonlinear equations. This method has been shown to effectively, easily, and accurately solve a large class of nonlinear problems with component converging rapidly to accurate solutions.

Differential-difference equations (DDEs) have been the focus of many nonlinear studies. DDEs describe many important phenomena and dynamical processes in many different fields, such as particle vibrations in lattices, currents in electrical networks, pulses in biological chains, and so on. DDEs play important role in the study of modern physics and also play a crucial role in numerical simulations of nonlinear partial differential equations (NLPDEs), queueing problems, and discretization in solid state and quantum physics. At the same time, finding exact solutions of DDEs is extremely important in mathematical physics.

On the other hand, in order to find directly exact solutions to DDEs, some methods [1626] for solving nonlinear differential equations are applied to DDEs. For example, Dehghan 
and Shakeri [16] have extended successfully multilinear variable separation approach to special DDEs. Baldwin et al. [26], Wang et al. [27] have applied homotopy analysis method (HAM) to DDEs. Dai and Zhang [28] have given a Jacobian elliptic function expansion method to solve the doubly periodic traveling wave solutions and kink-type tanh solitary solutions to some DDEs. There also have been some methods for nonlinear DDEs, such as Backlund transformation [29, 30], Hirota method [31, 32], Darboux transformation [33], and Adomian decomposition method [34].

\section{He's variational iteration method}

Now, to illustrate the basic concept of He's variational iteration method, we consider the following general nonlinear differential equation given in the form

$$
L u(t)+N u(t)=g(t),
$$

where $L$ is a linear operator, $N$ is a nonlinear operator, and $g(t)$ is a known analytical function. We can construct a correction functional according to the variational method as

$$
u_{n+1}(t)=u_{n}(t)+\int_{0}^{t} \lambda\left(L u_{n}(\xi)+N \tilde{u}_{n}(\xi)-g(\xi)\right) d \xi
$$

where $\lambda$ is a general Lagrange multiplier, which can be identified optimally via variational theory, the subscript $n$ denotes the $n$th approximation, and $\tilde{u}_{n}$ is considered as a restricted variation, namely $\delta \widetilde{u}_{n}=0$.

In the following example, we will illustrate the usefulness and effectiveness of the proposed technique.

\section{Application to Volterra equation}

Consider the following Volterra equation:

$$
\frac{d u_{n}}{d t}=u_{n}\left(u_{n+1}-u_{n-1}\right)
$$

with the initial condition

$$
u_{n}(0)=n,
$$

whose exact solution can be written as

$$
u_{n}(t)=\frac{n}{1-2 t}
$$

We apply variational iteration method to the discussed problem. Using He's variational iteration method, the correction functional can be written in the form

$$
u_{n, m+1}(t)=u_{n, m}(t)+\int_{0}^{t} \lambda(s)\left\{\frac{d u_{n, m}(s)}{d s}-\left(u_{n, m}(s)\right)\left(u_{n+1, m}(s)-u_{n-1, m}(s)\right)\right\} d s .
$$


The stationary conditions

$$
\begin{gathered}
1+\lambda=0, \\
\lambda^{\prime}=0
\end{gathered}
$$

follow immediately. This in turn gives

$$
\lambda=-1
$$

Substituting this value of the Lagrange multiplier $\lambda=-1$ into the functional (3.2) gives the iteration formula

$$
u_{n, m+1}(t)=u_{n, m}(t)-\int_{0}^{t}\left\{\frac{d u_{n, m}(s)}{d s}-\left(u_{n, m}(s)\right)\left(u_{n+1, m}(s)-u_{n-1, m}(s)\right)\right\} d s .
$$

We can start with $u_{n, 0}=n$, and we obtain the following successive approximations:

$$
\begin{gathered}
u_{n, 0}(t)=n, \\
u_{n, 1}(t)=n+2 n t, \\
u_{n, 2}(t)=n+2 n t+4 n t^{2}, \\
u_{n, 3}(t)=n+2 n t+4 n t^{2}+8 n t^{3}, \\
u_{n, 4}(t)=n+2 n t+4 n t^{2}+8 n t^{3}+16 n t^{4} .
\end{gathered}
$$

Hence, the solution series in general gives

$$
\begin{aligned}
& u_{n}(t)=n+2 n t+4 n t^{2}+8 n t^{3}+16 n t^{4} \ldots \\
& u_{n}(t)=n\left(1+2 t+4 t^{2}+8 t^{3}+16 t^{4} \ldots\right) .
\end{aligned}
$$

The closed form of the series (3.8) is $u_{n}(t)=n /(1-2 t)$ which gives exact solution of problem.

\section{Application to mKDV lattice equation}

Consider the following discretized mKDV lattice equation:

$$
\frac{d u_{n}}{d t}=\left(1-u_{n}^{2}\right)\left(u_{n+1}-u_{n-1}\right)
$$

with the initial condition

$$
u_{n}(0)=\tanh (k) \tanh (k n)
$$

We apply variational iteration method to the discussed problem. Using He's variational iteration method, the correction functional can be written in the form

$$
u_{n, m+1}(t)=u_{n, m}(t)+\int_{0}^{t} \lambda(s)\left\{\frac{d u_{n, m}(s)}{d s}-\left(1-u_{n, m}^{2}(s)\right)\left(u_{n+1, m}(s)-u_{n-1, m}(s)\right)\right\} d s .
$$


The stationary conditions

$$
\begin{gathered}
1+\lambda=0, \\
\lambda^{\prime}=0
\end{gathered}
$$

follow immediately. This in turn gives

$$
\lambda=-1
$$

Substituting this value of the Lagrange multiplier $\lambda=-1$ into the functional (4.1) gives the iteration formula

$$
u_{n, m+1}(t)=u_{n, m}(t)-\int_{0}^{t}\left\{\frac{d u_{n, m}(s)}{d s}-\left(1-u_{n, m}^{2}(s)\right)\left(u_{n+1, m}(s)-u_{n-1, m}(s)\right)\right\} d s .
$$

We can start with $u_{n, 0}=\tanh (k) \tanh (k n)$, and we obtain the following successive approximations:

$$
\begin{aligned}
& u_{n, 0}(t)=\tanh (k) \tanh (k n), \\
& u_{n, 1}(t)=\tanh (k) \tanh (k n) \\
& +[\tanh (k)(\tanh (k(n+1))-\tanh (k(n-1))) \\
& \left.-\tanh ^{2}(k) \tanh ^{2}(k n)(\tanh (k)(\tanh (k(n+1))-\tanh (k(n-1))))\right] t, \\
& u_{n, 2}(t)=\tanh (k) \tanh (k n) \\
& +[\tanh (k)(\tanh (k(n+1))-\tanh (k(n-1))) \\
& \left.-\tanh ^{2}(k) \tanh ^{2}(k n)(\tanh (k)(\tanh (k(n+1))-\tanh (k(n-1))))\right] t \\
& +[\tanh (k) \tanh (k(n+2))-2 \tanh (k) \tanh (k n) \\
& -\tanh ^{2}(k) \tanh ^{2}(k(n+1))(\tanh (k) \tanh (k(n+2)))-\tanh (k) \tanh (k n) \\
& +\tanh (k) \tanh (k(n-2))+\tanh ^{2}(k) \tanh ^{2}(k(n-1))(\tanh (k) \tanh (k n)) \\
& -\tanh (k) \tanh (k(n-2))-2 \tanh (k) \tanh (k n) \tanh (k) \tanh (k(n+1)) \\
& -\tanh (k) \tanh (k(n-1)) \tanh (k) \tanh (k(n+1))-\tanh (k) \tanh (k(n-1)) \\
& -\tanh ^{2}(k) \tanh ^{2}(k n) \tanh (k) \tanh (k(n+1))-\tanh (k) \tanh (k(n-1)) \\
& -\tanh ^{2}(k) \tanh ^{2}(k n)(\tanh (k) \tanh (k(n+2))-2 \tanh (k) \tanh (k n) \\
& \left.-\tanh ^{2}(k) \tanh ^{2}(k(n+1))\right) \\
& \times(\tanh (k) \tanh (k(n+2))-\tanh (k) \tanh (k n))+\tanh (k) \tanh (k(n-2)) \\
& \left.+\tanh ^{2}(k) \tanh ^{2}(k(n-1)) \tanh (k) \tanh (k n)-\tanh (k) \tanh (k(n-2))\right] 0.5 t^{2} .
\end{aligned}
$$

The other components of $u_{n, m}(t)$ can be generated in a similar way. Generally speaking, it is possible to calculate more components via some calculation software such as Maple to improve the accuracy of the approximate solutions. 
Table 1: For constant $k=0.1$, and time $t=0.5$.

\begin{tabular}{cccl}
\hline$n$ & $\mathrm{ADM}-u_{6}$ & $\mathrm{VIM}-u_{2}$ & Absolute error \\
\hline-25 & $-0,09804197166$ & $-0,09804373331$ & 0.00000176165 \\
-15 & $-0,08824837298$ & $-0,08825728153$ & 0,00000890855 \\
-5 & $-0,03789706610$ & $-0,03788612415$ & 0,00001094195 \\
0 & 0,009900946992 & 0,009933709149 & 0,000032762157 \\
5 & 0,05350310282 & 0,05351081023 & 0,00000770741 \\
15 & 0,091855587327 & 0,09184745591 & 0,00000813142 \\
25 & 0,09857365542 & 0,09857205219 & 0,00000160323 \\
\hline
\end{tabular}

Table 2: For constant $k=0.1$, and time $t=1.5$.

\begin{tabular}{cccc}
\hline$n$ & $\mathrm{ADM}-u_{6}$ & $\mathrm{VIM}-u_{2}$ & Absolute error \\
\hline-25 & $-0,09725516662$ & $-0,09730760788$ & 0,00005244126 \\
-15 & $-0,083118934180$ & $-0,08337156677$ & 0,00025263259 \\
-5 & $-0,01977150813$ & $-0,01938285277$ & 0,00038865536 \\
0 & 0,02894478018 & 0,02890112744 & 0,00004365274 \\
5 & 0,06613063122 & 0,06625691101 & 0,00012627979 \\
15 & 0,09435553904 & 0,09414208992 & 0,00021344912 \\
25 & 0,09893218337 & 0,09889256453 & 0,00003961884 \\
\hline
\end{tabular}

In order to verify numerically whether the proposed methodology leads to high accuracy, we evaluate the numerical solutions using only second-order approximation and compared it with Adomian decomposition solution (ADM) using six-term approximation [34]. Tables 1 and 2 show the absolute errors between ADM- $u_{6}$ and numerical solution (VIM- $u_{2}$ ) of (16a) with initial condition (16b).

Tables 1 and 2 show that the numerical approximate solution has a high degree of accuracy. As we know, the more terms added to the approximate solution, the more accurate it will be. Although we only considered second-order approximation, it achieves a high level of accuracy.

\section{Conclusion}

In this paper, by the variational iteration method, firstly, we obtain the exact solution of Volterra equation. Secondly, we obtain the approximate solution of mKDV lattice equation. The method is extremely simple, easy to use, and is very accurate for solving nonlinear differential-difference equation. Also, the method is a powerful tool to search for solutions of various linear/nonlinear problems. This variational iteration method will become a much more interesting method to solve nonlinear DDEs in science and engineering.

\section{Acknowledgment}

The author thanks The Scientific and Technological Research Council of Turkey (TÜB İ TAK) for their financial support. 


\section{References}

[1] J.-H. He and X.-H. Wu, "Exp-function method for nonlinear wave equations," Chaos, Solitons $\mathcal{E}$ Fractals, vol. 30, no. 3, pp. 700-708, 2006.

[2] J.-H. He, "Homotopy perturbation method: a new nonlinear analytical technique," Applied Mathematics and Computation, vol. 135, no. 1, pp. 73-79, 2003.

[3] J.-H. He, "The homotopy perturbation method nonlinear oscillators with discontinuities," Applied Mathematics and Computation, vol. 151, no. 1, pp. 287-292, 2004.

[4] J.-H. He, "Application of homotopy perturbation method to nonlinear wave equations," Chaos, Solitons E Fractals, vol. 26, no. 3, pp. 695-700, 2005.

[5] J.-H. He, "Homotopy perturbation method for bifurcation of nonlinear problems," International Journal of Nonlinear Sciences and Numerical Simulation, vol. 6, no. 2, pp. 207-208, 2005.

[6] J.-H. He, "Homotopy perturbation method for solving boundary value problems," Physics Letters A, vol. 350, no. 1-2, pp. 87-88, 2006.

[7] T. Öziş and A. Yıldırım, "A note on He's homotopy perturbation method for van der Pol oscillator with very strong nonlinearity," Chaos, Solitons E Fractals, vol. 34, no. 3, pp. 989-991, 2007.

[8] T. Öziş and A. Yıldırım, "A comparative study of He's homotopy perturbation method for determining frequency-amplitude relation of a nonlinear oscillator with discontinuities," International Journal of Nonlinear Sciences and Numerical Simulation, vol. 8, no. 2, pp. 243-248, 2007.

[9] T. Öziş and A. Yıldırım, "Traveling wave solution of Korteweg-de vries equation using He's homotopy prturbation method," International Journal of Nonlinear Sciences and Numerical Simulation, vol. 8, no. 2, pp. 239-242, 2007.

[10] T. Öziş and A. Yıldırım, "Determination of periodic solution for a $u^{1 / 3}$ force by He's modified Lindstedt-Poincaré method," Journal of Sound and Vibration, vol. 301, no. 1-2, pp. 415-419, 2007.

[11] A. Yildırım and T. Öziş, "Solutions of singular IVPs of Lane-Emden type by homotopy perturbation method," Physics Letters A, vol. 369, no. 1-2, pp. 70-76, 2007.

[12] J.-H. He, "Variational iteration method-a kind of non-linear analytical technique: some examples," International Journal of Non-Linear Mechanics, vol. 34, no. 4, pp. 699-708, 1999.

[13] J.-H. He, "Variational iteration method for autonomous ordinary differential systems," Applied Mathematics and Computation, vol. 114, no. 2-3, pp. 115-123, 2000.

[14] J.-H. He and X.-H. Wu, "Construction of solitary solution and compacton-like solution by variational iteration method," Chaos, Solitons E Fractals, vol. 29, no. 1, pp. 108-113, 2006.

[15] T. Öziş and A. Yıldırım, "A study of nonlinear oscillators with $u^{1 / 3}$ force by He's variational iteration method," Journal of Sound and Vibration, vol. 306, no. 1-2, pp. 372-376, 2007.

[16] M. Dehghan and F. Shakeri, "Application of He's variational iteration method for solving the Cauchy reaction-diffusion problem," Journal of Computational and Applied Mathematics, vol. 214, no. 2, pp. 435446, 2008.

[17] F. Shakeri and M. Dehghan, "Solution of delay differential equations via a homotopy perturbation method," Mathematical and Computer Modelling. In press.

[18] M. Dehghan and F. Shakeri, "Approximate solution of a differential equation arising in astrophysics using the variational iteration method," New Astronomy, vol. 13, no. 1, pp. 53-59, 2008.

[19] F. Shakeri and M. Dehghan, "Numerical solution of a biological population model using He's variational iteration method," Computers $\mathcal{E}$ Mathematics with Applications, vol. 54, no. 7-8, pp. 11971209, 2007.

[20] F. Shakeri and M. Dehghan, "Numerical solution of the Klein-Gordon equation via He's variational iteration method," Nonlinear Dynamics, vol. 51, no. 1-2, pp. 89-97, 2008.

[21] M. Tatari and M. Dehghan, "On the convergence of He's variational iteration method," Journal of Computational and Applied Mathematics, vol. 207, no. 1, pp. 121-128, 2007.

[22] M. Tatari and M. Dehghan, "Solution of problems in calculus of variations via He's variational iteration method," Physics Letters A, vol. 362, no. 5-6, pp. 401-406, 2007.

[23] M. Dehghan and M. Tatari, "The use of He's variational iteration method for solving a Fokker-Planck equation," Physica Scripta, vol. 74, no. 3, pp. 310-316, 2006.

[24] M. Dehghan and M. Tatari, "Identifying an unknown function in a parabolic equation with overspecified data via He's variational iteration method," Chaos, Solitons E Fractals, vol. 36, no. 1, pp. 157-166, 2008. 
[25] M. Tatari and M. Dehghan, "He's variational iteration method for computing a control parameter in a semi-linear inverse parabolic equation," Chaos, Solitons E Fractals, vol. 33, no. 2, pp. 671-677, 2007.

[26] D. Baldwin, Ü. Göktaş, and W. Hereman, "Symbolic computation of hyperbolic tangent solutions for nonlinear differential-difference equations," Computer Physics Communications, vol. 162, no. 3, pp. 203217, 2004.

[27] Z. Wang, L. Zou, and H. Zhang, "Applying homotopy analysis method for solving differentialdifference equation," Physics Letters A, vol. 369, no. 1-2, pp. 77-84, 2007.

[28] C. Dai and J. Zhang, "Jacobian elliptic function method for nonlinear differential-difference equations," Chaos, Solitons \& Fractals, vol. 27, no. 4, pp. 1042-1047, 2006.

[29] M. Sun, S. Deng, and D. Chen, "The Bäcklund transformation and novel solutions for the Toda lattice," Chaos, Solitons E Fractals, vol. 23, no. 4, pp. 1169-1175, 2005.

[30] R. Hirota, X.-B. Hu, and X.-Y. Tang, "A vector potential KdV equation and vector Ito equation: soliton solutions, bilinear Bäcklund transformations and Lax pairs," Journal of Mathematical Analysis and Applications, vol. 288, no. 1, pp. 326-348, 2003.

[31] X.-B. Hu and Y.-T. Wu, "Application of the Hirota bilinear formalism to a new integrable differentialdifference equation," Physics Letters A, vol. 246, no. 6, pp. 523-529, 1998.

[32] X.-B. Hu and H.-W. Tam, "Application of Hirota's bilinear formalism to a two-dimensional lattice by Leznov," Physics Letters A, vol. 276, no. 1-4, pp. 65-72, 2000.

[33] M. Mañas, A. Doliwa, and P. M. Santini, "Darboux transformations for multidimensional quadrilateral lattices. I," Physics Letters A, vol. 232, no. 1-2, pp. 99-105, 1997.

[34] L. Wu, L. D. Xie, and J. F. Zhang, "Adomian decomposition method for nonlinear differentialdifference equations," Communications in Nonlinear Science and Numerical Simulation. In press. 


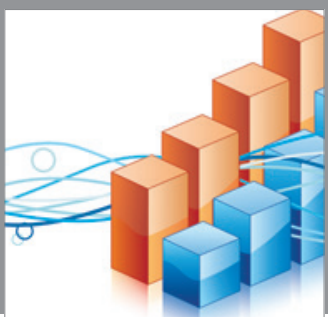

Advances in

Operations Research

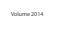

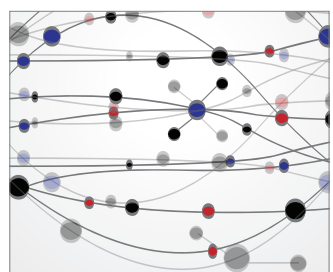

\section{The Scientific} World Journal
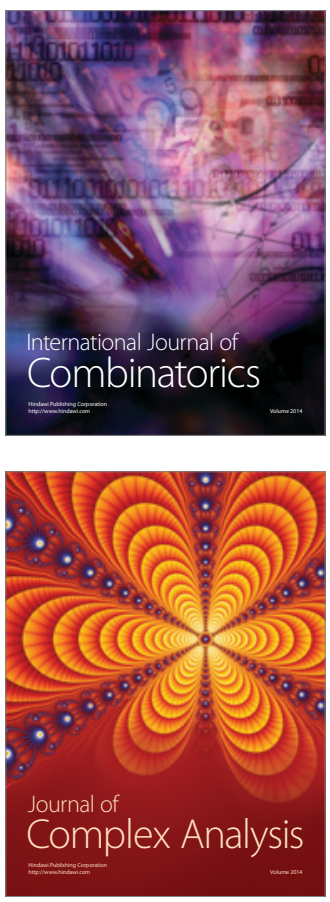

International Journal of

Mathematics and

Mathematical

Sciences
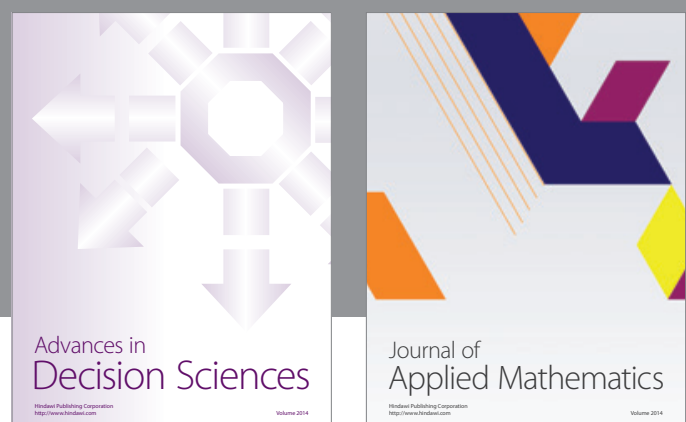

Journal of

Applied Mathematics
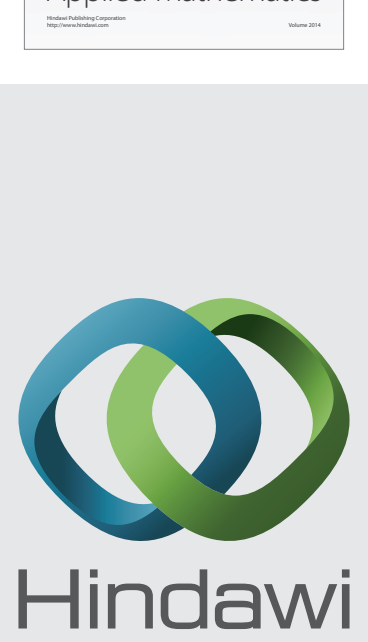

Submit your manuscripts at http://www.hindawi.com
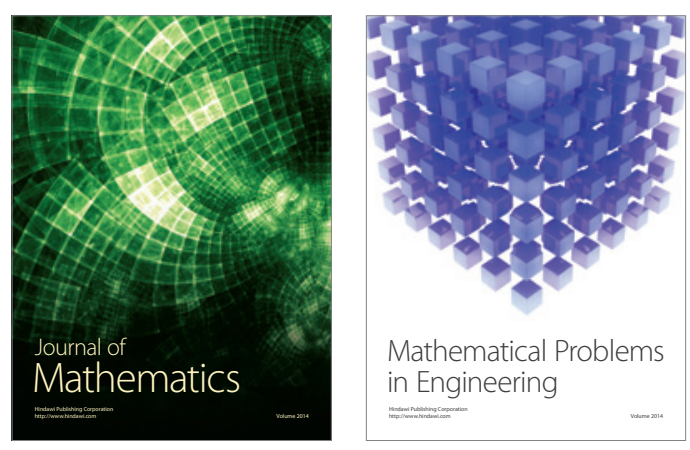

Mathematical Problems in Engineering
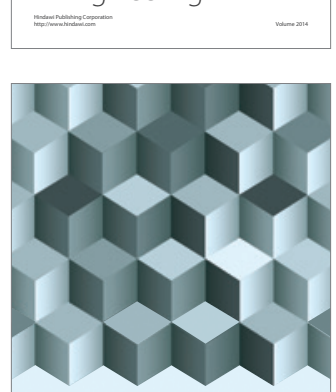

Journal of

Function Spaces
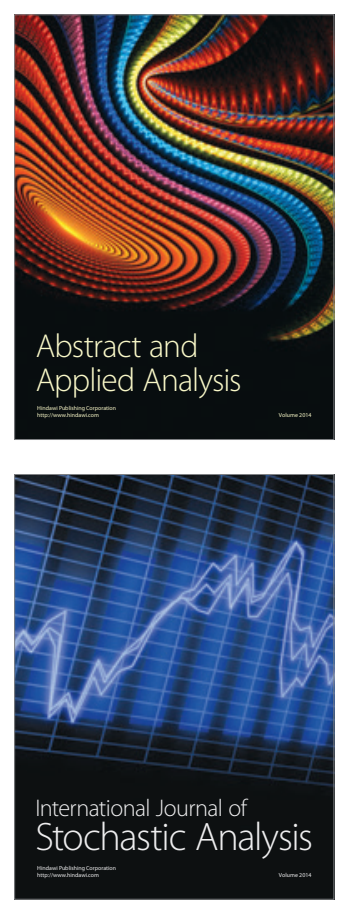

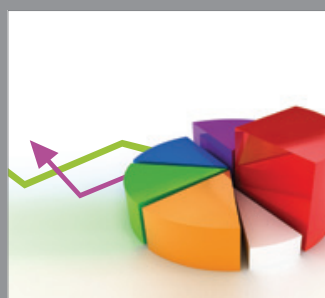

ournal of

Probability and Statistics

Promensencen
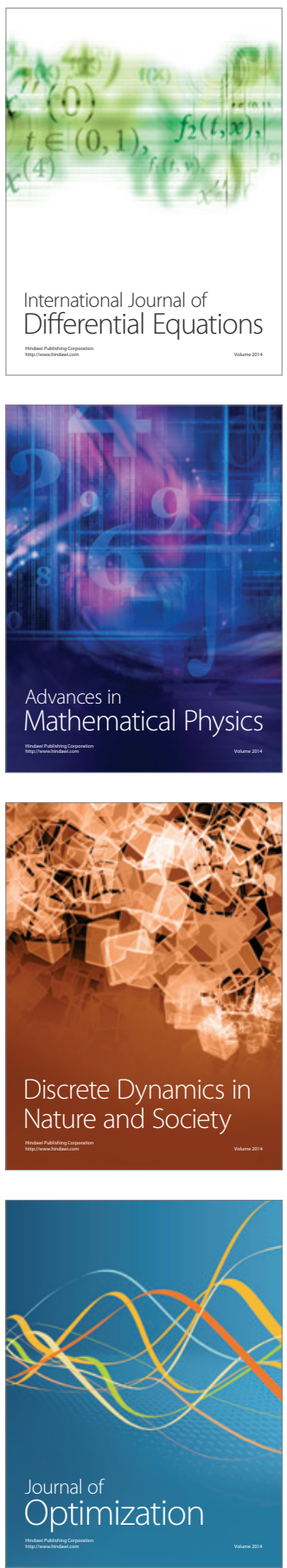